\title{
Localização e freqüência das alterações de campo visual no glaucoma primário de ângulo aberto na estratégia SITA (Swedish Interactive Threshold Algorithm)
}

\author{
Location and frequency of visual field defects as measured by SITA (Swedish Interacti- \\ ve Threshold Algorithm) strategy in primary open angle glaucoma
}

\author{
Vanessa Cristina Ruegger Nascimento ${ }^{1}$ \\ Niro Kasahara ${ }^{2}$ \\ Ralph Cohen ${ }^{3}$ \\ Geraldo Vicente de Almeida ${ }^{4}$ \\ Carmo Mandia $\mathbf{J r}^{5}$ \\ Maurício Della Paolera ${ }^{6}$
}

Trabalho realizado na Faculdade de Ciências Médicas da Santa Casa de Misericórdia de São Paulo.

Fellow do Setor de Córnea e Doenças Externas da Faculdade de Ciências Médicas da Santa Casa de Misericórdia de São Paulo. São Paulo (SP).

Assistente do Setor de Glaucoma da Faculdade de Ciências Médicas da Santa Casa de Misericórdia de São Paulo. São Paulo (SP)

Professor Doutor, Chefe do Setor de Glaucoma da Faculdade de Ciências Médicas da Santa Casa de Misericórdia de São Paulo. São Paulo (SP).

${ }^{4}$ Professor Doutor, Diretor do Departamento de Oftalmologia da Faculdade de Ciências Médicas da Santa Casa de Misericórdia de São Paulo. São Paulo (SP).

Assistente do Setor de Glaucoma da Faculdade de Ciências Médicas da Santa Casa de Misericórdia de São Paulo. São Paulo (SP).

${ }^{6}$ Assistente do Setor de Glaucoma da Faculdade de Ciências Médicas da Santa Casa de Misericórdia de São Paulo. São Paulo (SP).

Endereço para correspondência: Rua Santo André, 430/21 - Santo André (SP) CEP 09020-230

E-mail: vanessaruegger@uol.com.br

Recebido para publicação em 20.08.2004

Versão revisada recebida em 28.01.2005

Aprovação em 10.05.2005

Nota Editorial: Depois de concluída a análise do artigo sob sigilo editorial e com a anuência do Dr. Riuitiro Yamane sobre a divulgação de seu nome como revisor, agradecemos sua participação neste processo.

\begin{tabular}{|l|}
\hline \multicolumn{1}{c|}{ RESUMO } \\
\hline Objetivo: Caracterizar os defeitos de campo visual nos exames realizados \\
com o perímetro Humphrey ${ }^{\circledR}$, estratégia SITA - Swedish Interactive \\
Threshold Algorithm - segundo sua freqüência e localização em pacientes \\
com glaucoma primário de ângulo aberto (GPAA). Métodos: Realizamos \\
análise retrospectiva de 6.200 prontuários. Avaliamos o exame de campo \\
visual dos pacientes que preenchiam os critérios de inclusão e os dividi- \\
mos em glaucoma leve, moderado e grave de acordo com a classificação \\
de Hodapp. Definimos os seguintes defeitos glaucomatosos: degrau \\
nasal, escotoma paracentral, escotoma arqueado, escotoma Seidel, defeito \\
temporal em cunha, diminuição generalizada de sensibilidade, escotoma \\
anular e campo tubular. Utilizamos diagrama que correlaciona os pontos \\
do campo visual comas regiões do disco óptico e avaliamos as regiões mais \\
acometidas de acordo com a densidade de pontos comprometidos. A \\
análise estatística foi realizada aplicando-se o teste $t$ de Student. Resultados: \\
Selecionamos 152 pacientes com idade média de $66,5 \pm 9,6 \%$, sendo $59,9 \%$ \\
do sexo feminino. No glaucoma leve o defeito campimétrico mais freqüente \\
foi o degrau nasal superior, seguido respectivamente pelo escotoma \\
paracentral e degrau nasal inferior. A diminuição generalizada de sensibi- \\
lidade, apesar de rara, ocorreu em $7,8 \%$ desses pacientes. A região superior \\
foi mais acometida que a inferior. Conclusão: O degrau nasal superior e o \\
escotoma paracentral foram os defeitos mais freqüentemente observados \\
no glaucoma primário de ângulo aberto em fase inicial pela estratégia SITA, \\
e o hemicampo superior foi a região mais acometida. A diminuição gene- \\
ralizada de sensibilidade foi defeito glaucomatoso precoce. \\
\hline
\end{tabular}

Descritores: Glaucoma/diagnóstico; Perimetria/métodos; Limiar sensorial; Campos visuais; Sensibilidade e especificidade

\section{INTRODUÇÃOO}

Glaucoma primário de ângulo aberto (GPAA) é uma importante causa de cegueira no mundo ${ }^{(1)}$. Geralmente os pacientes são assintomáticos, até que ocorram danos anatômicos e funcionais tardios do nervo óptico ${ }^{(2)}$. A perimetria computadorizada permite a avaliação funcional do nervo óptico e é utilizada para diagnosticar, determinar a gravidade da lesão e acompanhar a evolução da doença ${ }^{(1-2)}$. Não permite diagnóstico precoce, pois a perda de fibras da camada de fibras nervosas da retina precede o aparecimento de defeitos campimétricos visuais ${ }^{(3-4)}$. Alterações na perimetria computadori- 
zada são observadas quando cerca de $40 \%$ das fibras ganglionares já foram lesadas ${ }^{(5)}$.

O defeito inicial mais freqüente é o degrau nasal, que pode ou não estar acompanhado de escotoma paracentral ${ }^{(6-7)}$. Aquele evolui lentamente em extensão e profundidade, ou com a formação de um novo escotoma. Os defeitos campimétricos incluem o degrau nasal, escotoma arqueado, escotoma paracentral, escotoma de Seidel (escotoma paracentral adjacente à mancha cega), defeito temporal em cunha e diminuição generalizada (difusa) de sensibilidade. Entretanto, tais defeitos foram descritos para a perimetria cinética de Goldmann ${ }^{(2)}$.

A perimetria computadorizada permite a pesquisa do limiar retínico de sensibilidade em vários pontos dentro dos $30^{\circ}$ graus centrais do campo visual. Um estímulo no tamanho III de Goldmann é apresentado aleatoriamente e com intensidades variadas, conforme a estratégia utilizada pelo programa previamente estabelecido. A estratégia denominada SITA ("Swedish Interactive Threshold Algorithm") utilizada no perímetro computadorizado de Humphrey ${ }^{\circledR}$ foi desenvolvida com a finalidade de diminuir a duração do exame. Na literatura encontramos estudos que demonstraram que este novo algoritmo apresentou menor tempo de duração que a estratégia convencional ("full threshold"), e constataram que esse novo exame apresenta desempenho semelhante na identificação de defeito de campo glaucomatoso em relação à estratégia convencional $^{(8-9)}$. Em outros trabalhos, os mesmos autores concluem que a estratégia SITA pode substituir a estratégia "full threshold" como teste padrão na avaliação perimétrica do paciente glaucomatoso ${ }^{(10)}$. Estudos demonstraram sensibilidade da estratégia SITA superior a 95\%, não havendo diferença em relação ao programa "full threshold" (11-12).

O objetivo do nosso estudo foi caracterizar os defeitos de campo visual nos exames realizados com o perímetro Humphrey ${ }^{\circledast}$, estratégia SITA, segundo sua freqüência e localização em pacientes com GPAA.

\section{MÉTODOS}

Realizamos uma análise retrospectiva de 6.200 prontuários do Ambulatório de Glaucoma do Departamento de Oftalmologia da Irmandade da Santa Casa de Misericórdia de São Paulo, arquivados entre janeiro de 1993 e março de 2003. O Comitê de Ética da Irmandade da Santa Casa de Misericórdia de São Paulo aprovou o estudo. Selecionamos os prontuários de pacientes que preenchiam os seguintes critérios de inclusão:

- Pacientes com GPAA, definidos pelo aspecto da escavação do disco óptico (escavação >0,5 ou perda localizada da rima), gonioscopia com seio camerular aberto, e pressão intra-ocular $>21 \mathrm{mmHg}$.

- Acuidade visual (AV) $\geq 20 / 40$ (tabela de Snellen) com a melhor correção óptica.

- Dois ou mais campos visuais confiáveis realizados com o programa central 24-2 estratégia SITA.

- Ametropia entre -4,00 DE e +6,00 DE, e $\leq-3,00 \mathrm{DC}$.
Além dos pacientes que não preenchiam os critérios de inclusão, também excluímos pacientes com glaucoma grave que apresentavam colapso dos índices estatísticos, isto é, gráfico de "pattern deviation" sem um único ponto com $\mathrm{p}<5 \%$, ou $\mathrm{PSD}<5 \%$ (ou dentro do normal) ${ }^{(13)}$. Dados referentes à $\mathrm{AV}$ (com a melhor correção óptica), raça, sexo e idade foram coletados dos prontuários.

Selecionamos 152 pacientes que preenchiam os critérios de inclusão. A idade dos pacientes variou entre 35 e 87 anos, com média de 66,5 $\pm 9,6$ anos; a acuidade visual, com correção refracional, medida por meio da tabela de Snellen, variou entre 0,5 e 1,0 , com média de $0,7 \pm 0,2$. A maioria dos pacientes eram brancos $(53,1 \%)$ e do sexo feminino $(59,9 \%)$, conforme observado na tabela 1 .

Todos os exames foram realizados por um técnico treinado, com o campímetro Humphrey ${ }^{\circledR}$ (HVFA II, Zeiss-Humphrey Systems, Dublin, CA, EUA), utilizando a correção óptica adequada para cada paciente. Cada exame de campo visual foi analisado por dois examinadores experientes que definiram os defeitos alistados a seguir:

- Degrau nasal: presença de três ou mais pontos adjacentes com $\mathrm{p}<5 \%$ e um ponto com $\mathrm{p}<1 \%$ ao longo do meridiano horizontal no hemicampo nasal; que pode ser superior (DNS) ou inferior (DNI) no gráfico de "pattern deviation".

- Escotoma paracentral (EPC): presença de três pontos adjacentes com $\mathrm{p}<5 \%$ e um deles com $\mathrm{p}<1 \%$, não adjacente à mancha cega, em qualquer região dos $30^{\circ}$ centrais do campo visual, exceto na região temporal, no gráfico de "pattern deviation".

- Escotoma arqueado: presença de cinco ou mais pontos com $\mathrm{p}<5 \%$ e um ponto com $\mathrm{p}<1 \%$, no gráfico de "pattern deviation", não adjacentes à mancha cega, não compreendidos no feixe papilo-macular e feixe nasal, apresentando a conformação de arco do gráfico da escala cinza, e não se estendendo além do hemicampo oposto. Pode ser superior (EAS) ou inferior (EAI).

- Escotoma Seidel (escotoma em vírgula): presença de três ou mais pontos com $\mathrm{p}<5 \%$ e um ponto com $\mathrm{p}<1 \%$ adjacente à mancha cega, excetuando os quatro pontos tem-

\begin{tabular}{|lc|}
\hline \multicolumn{2}{|c|}{ Tabela 1. Características demográficas da amostra } \\
Características da população & $\%$ ou $\mathbf{X} \pm \mathbf{D P}$ \\
\hline Idade (anos) & $66,5 \pm 9,6$ \\
AV* & $0,7 \pm 0,2$ \\
Sexo & \\
Feminino & $59,9 \%$ \\
Masculino & $40,1 \%$ \\
Raça & $53,1 \%$ \\
Branca & $22,8 \%$ \\
Parda & $24,1 \%$ \\
Negra & \\
*AV=Acuidade visual aferida coma tabela de Snellen, ea melhor correçãoóptica. \\
Fonte: Departamento de Oftalmologia-Glaucoma da Irmandade da Santa Casa \\
de Misericórdia de São Paulo, janeiro/1993 a março/2003. \\
\hline
\end{tabular}


porais do campo visual no gráfico de "pattern deviation". Pode ser superior (ESS) ou inferior (ESI).

- Defeito temporal (DT): presença de três pontos com $\mathrm{p}<5 \%$ e um com $\mathrm{p}<1 \%$ na região temporal no gráfico de "pattern deviation".

- Diminuição generalizada de sensibilidade (DGS): MD (mean deviation) com $\mathrm{p}<5 \%$ e presença de $50 \%$ ou mais de pontos no gráfico de "total deviation" com $\mathrm{p}<5 \% \mathrm{em}$ pacientes com acuidade visual $\geq 20 / 25$, ou conforme indicado no GHT.

- Escotoma anular: formado pela união de dois escotomas arqueados, um superior e um inferior no gráfico de "pattern deviation".

- Campo tubular: diminuição concêntrica da sensibilidade em pontos além dos $10^{\circ}$ graus centrais do campo visual no gráfico de "pattern deviation".

Os exames de campo visual foram classificados em glaucoma inicial, moderado ou grave, de acordo com a classificação proposta na literatura existente ${ }^{(14)}$.

Após classificá-los, utilizamos um diagrama que relaciona os pontos do exame de campo visual a regiões da cabeça do nervo óptico ${ }^{(15)}$. Neste diagrama os autores determinaram a correspondência de cada ponto do campo visual com uma localização correspondente na retina e na rima neural. Os 52 pontos do campo visual foram divididos em seis setores, correspondendo às seis regiões da rima neural do disco óptico (Figura 1).

Contamos o número de pontos com $\mathrm{p}<5 \%$ no gráfico do pattern deviation em cada uma das seis regiões e, para cada região, foi calculada a densidade de pontos comprometidos $(\mathrm{p}<5 \%)$ de acordo com a fórmula: densidade $=\mathrm{n}^{\circ} \mathrm{e}$ de pontos com $\mathrm{p}<5 \%$ em cada região/n으 total de pontos em cada região.

A comparação da diferença da densidade de pontos entre regiões correspondentes do hemicampo superior e inferior foi feita utilizando-se o teste $t$ de Student para amostras pareadas, com significância a $5 \%(\alpha=0,05 \%)$.

\section{RESULTADOS}

Selecionamos 152 pacientes que preenchiam os critérios de inclusão anteriormente citados, provendo uma amostra de 152 exames de campo visual. Os dados demográficos estão apresentados na tabela 1 .

A análise dos defeitos, segundo a gravidade do glaucoma mostrou que o degrau nasal superior foi encontrado em maior frequiência no glaucoma inicial $(33,3 \%)$ e moderado $(47,6 \%)$ e o escotoma arqueado superior no glaucoma grave $(49,1 \%)$, (Tabela 2). A tabela 3 mostra a densidade dos pontos comprometidos em cada um dos seis setores do campo visual. Nos glaucomas iniciais o arco superior interno $(35,7 \%)$ foi mais acometido que $\mathrm{o}$ arco inferior interno $(21,4 \%$; $\mathrm{p}=0,01)$, e o arco superior externo $(31,6 \%)$ foi mais acometido que o arco inferior externo $(15,5 \% ; \mathrm{p} \leq 0,001)$.

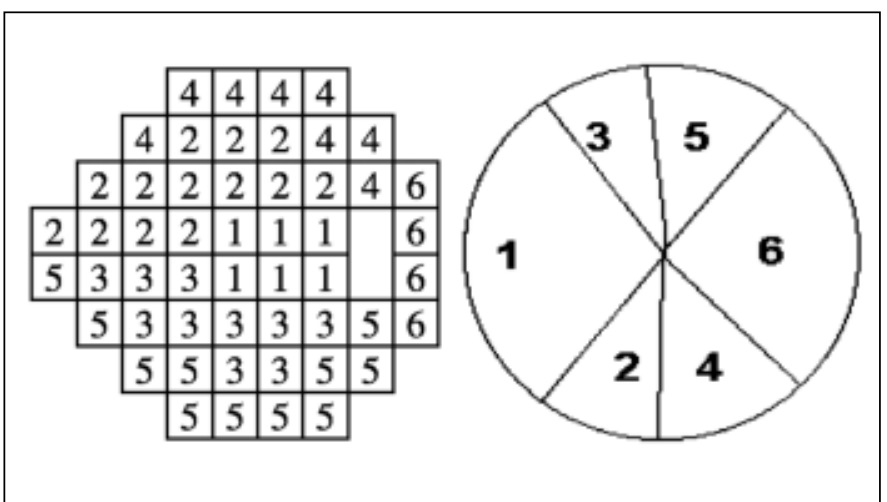

Figura 1 - Correspondência entre os pontos do exame de perimetria computadorizada (programa central 24-2) com 6 setores do disco óptico. Os números correspondem às seguintes áreas determinadas pelos examinadores: № 1 - feixe papilo-macular $\left(40-311^{\circ}\right)$; № 2 - Arco superior interno $\left(271-310^{\circ}\right)$; № 3 - Arco inferior interno (41-80 $)$; № 4 - Arco superior externo $\left(231-270^{\circ}\right)$; № 5 - Arco inferior externo $\left(81-120^{\circ}\right)$; № 6 - Feixe nasal $\left(121-230^{\circ}\right)$

\begin{tabular}{|c|c|c|c|c|}
\hline $\begin{array}{l}\text { Defeito no exame } \\
\text { de campo visual* }\end{array}$ & $\begin{array}{l}\text { Glaucoma inicial }^{\star *} \\
\qquad(n=51)\end{array}$ & $\begin{array}{l}\text { Glaucoma moderado** } \\
\qquad(n=42)\end{array}$ & $\begin{array}{l}\text { Glaucoma grave }{ }^{\star *} \\
\qquad(n=59)\end{array}$ & $\begin{array}{c}\text { Total } \\
(n=152)\end{array}$ \\
\hline Degrau nasal superior & $33,3 \%$ & $47,6 \%$ & $40,6 \%$ & $40,1 \%$ \\
\hline Degrau nasal inferior & $19,6 \%$ & $28,6 \%$ & $35,6 \%$ & $28,3 \%$ \\
\hline Escotoma arqueado superior & $5,9 \%$ & $35,7 \%$ & $49,1 \%$ & $30,9 \%$ \\
\hline Escotoma arqueado inferior & & $14,3 \%$ & $23,7 \%$ & $13,2 \%$ \\
\hline Escotoma paracentral & $23,5 \%$ & $11,9 \%$ & $6,8 \%$ & $13,8 \%$ \\
\hline Escotoma Seidel superior & $15,7 \%$ & $11,9 \%$ & $6,8 \%$ & $11,2 \%$ \\
\hline Escotoma Seidel inferior & $9,8 \%$ & $7,1 \%$ & $6,8 \%$ & $7,9 \%$ \\
\hline Defeito temporal cunha & $2,0 \%$ & $11,9 \%$ & $8,5 \%$ & $7,2 \%$ \\
\hline Diminuição generalizada sensibilidade & $7,8 \%$ & $4,8 \%$ & & $3,9 \%$ \\
\hline Campo tubular & & & $16,9 \%$ & $6,6 \%$ \\
\hline Escotoma anular & & & $13,6 \%$ & $5,3 \%$ \\
\hline
\end{tabular}


Tabela 3. Porcentagem (\%) de no de pontos com $p<5 \%$ em cada região

\begin{tabular}{|c|c|c|c|}
\hline Setor do campo visual* & $\begin{array}{l}\text { Glaucoma inicial }^{\star *} \\
(n=51)\end{array}$ & $\begin{array}{l}\text { Glaucoma moderado** } \\
\qquad(n=42)\end{array}$ & $\begin{array}{l}\text { Glaucoma grave }{ }^{\star *} \\
\qquad(n=59)\end{array}$ \\
\hline Arco superior interno & $35,7( \pm 28,9)$ & $52,4( \pm 35,8)$ & $84,1( \pm 29,6)$ \\
\hline Arco inferior interno & $21,4( \pm 16,9)$ & $29,8( \pm 32,5)$ & $60,3( \pm 33,3)$ \\
\hline Arco inferior externo & $15,5( \pm 17,5)$ & $34,6( \pm 30,1)$ & $63,0( \pm 31,2)$ \\
\hline Feixe nasal & $18,9( \pm 31,0)$ & $25,6( \pm 34,2)$ & $35,6( \pm 34,8)$ \\
\hline
\end{tabular}

\section{DISCUSSÃO}

Os escotomas paracentral e arqueado, e o degrau nasal foram inicialmente descritos mediante a perimetria cinética de Goldmann. Muitos estudos clínicos, como o estudo de intervenção no glaucoma avançado (AGIS), resumem todos os dados do campo visual em um valor numérico único ${ }^{(16)}$. Entretanto, a interpretação do exame baseada na confiança em critérios estatísticos é perigosa em aplicações clínicas. Um observador experiente é capaz de reconhecer se as alterações nos resultados estatísticos do StatPac são determinadas por um defeito da camada de fibras nervosas da retina típico de glaucoma ou por qualquer artefato. Portanto, é fundamental conhecer os defeitos perimétricos característicos de glaucoma conforme observados nos gráficos da escala cinza, "total" e "pattern deviation" no impresso do exame.

Em nosso estudo, o degrau nasal superior foi o defeito mais encontrado no glaucoma leve, seguido pelo escotoma paracentral. Nossos resultados concordam com a observação de alguns autores que consideram o degrau nasal superior e o escotoma paracentral como lesões iniciais no glaucoma conforme observados pela perimetria de Goldmann ${ }^{(7)}$. Outros autores observaram que a distribuição dos defeitos precoces no campo visual em 214 olhos de pacientes com GPAA inicial revelou uma combinação de escotoma paracentral com defeito periférico em $75,2 \%{ }^{(17)}$. Nosso estudo difere de outros que demonstraram que os defeitos perimétricos mais freqüentemente detectados por meio da perimetria manual foram, em ordem decrescente: escotoma arqueado, degrau nasal, escotoma paracentral, escotoma Seidel, e defeito temporal em cunha, sendo a região superior mais acometida que a inferior ${ }^{(6)}$.

A diminuição generalizada de sensibilidade é um defeito pouco freqüente no glaucoma e pode ser considerado um defeito precoce, porém é inespecífico, pois pode estar presente em outras neuropatias ópticas ${ }^{(18)}$. Além disso, alterações de transparência, como opacidades corneais ou lenticulares, também podem ocasioná-la. Surpreendentemente, em nosso estudo observamos, no glaucoma leve, 7,8\% de freqüência deste defeito, que foi observado somente em pacientes com $A V \geq 20 / 25$, ausência de opacidades ópticas e exames realizados com correção óptica adequada. Dessa forma, a diminuição generalizada de sensibilidade está de fato relacionada ao glaucoma, sendo mais freqüente que o escotoma arqueado no glaucoma leve. Esse achado só terá valor se compatível com o aspecto do disco óptico e descartadas outras possíveis causas.

$\mathrm{O}$ defeito temporal em cunha foi muito raro no glaucoma leve, provavelmente porque as fibras mediais são as últimas a serem lesadas e sua incidência, mesmo no glaucoma moderado e grave, foi baixa. Essas fibras correspondem à rima do quadrante nasal do disco óptico. Analisando a importância do setor nasal do disco óptico no diagnóstico morfométrico do glaucoma, alguns autores, por meio de estereofoto colorida, constataram que quando comparada à avaliação das outras regiões do disco, a avaliação da região nasal tem menor importância em detectar o dano glaucomatoso do nervo óptico ${ }^{(19)}$. Entretanto, não sugerem excluir a região nasal do disco óptico na avaliação do glaucoma. Certamente, nos glaucomas avançados a rima nasal com frequiência é o que resta para ser avaliado. Nos pacientes com remanescente temporal do campo visual deve-se valorizar a sensibilidade destes pontos na avaliação funcional da progressão do glaucoma.

A análise setorial dos pontos deprimidos com $\mathrm{p}<5 \%$ nos glaucomas leves revelou maior densidade nos arcos superior (interno e externo), seguidos pelo arco inferior interno e feixe nasal. Nossos resultados concordam com a observação de outro estudo literário que avaliando 50 olhos de pacientes com GPAA e defeitos precoces com a perimetria de Goldmann, constataram que os defeitos no hemicampo superior eram duas vezes mais frequientes que os defeitos no hemicampo inferior ${ }^{(20)}$.

Os pontos correspondentes às fibras do feixe papilo-macular e do feixe nasal são relativamente preservadas nos glaucomas graves (densidade de 40,9\% e 33,6\%, respectivamente). Estes resultados concordam com a observação de que as fibras nasais e as fibras do feixe papilo-macular são as últimas a serem lesadas no glaucoma.

A análise dos índices globais derivados do StatPac, os gráficos de "total" e "pattern deviation" substituíram a avaliação morfo-qualitativa da perimetria manual para diagnóstico e avaliação da progressão da lesão glaucomatosa. Entretanto, os defeitos perimétricos evidenciados na escala cinza e confirmados por pontos deprimidos no "pattern deviation" são importantes para a interpretação do exame realizado com a estra- 
tégia SITA e, até que se torne comercialmente disponível em nosso meio o programa "Glaucoma Progression Analyses ${ }^{\circledR}$ ", a análise individual da sensibilidade para cada ponto do campo visual é o único meio de avaliar a progressão do defeito.

Nosso estudo foi retrospectivo e apresenta as limitações inerentes a estudos que usam dados de prontuários. Dentre a literatura pesquisada este foi o primeiro trabalho que estudou a morfologia dos defeitos campimétricos utilizando-se a perimetria computadorizada com a estratégia SITA. Este estudo ilustra de maneira clara os defeitos mais freqüentes e regiões mais acometidas no glaucoma, trazendo importantes implicações clínicas. Ao utilizar-se a estratégia SITA, o examinador deve estar atento às regiões correspondentes ao arco superior interno e externo, valorizando os pontos com $\mathrm{p}<5 \%$ como sugestivos de defeito glaucomatoso inicial.

\section{CONCLUSÃO}

1. O degrau nasal superior e o escotoma paracentral foram os defeitos mais freqüentemente observados no GPAA em fase inicial conforme determinado na perimetria computadorizada com a estratégia SITA.

2. A diminuição generalizada de sensibilidade foi defeito glaucomatoso precoce, mas pouco freqüente.

3. O hemicampo superior, cujos pontos correspondem à fibra da rima neural do quadrante inferior do disco óptico, foi a região mais acometida no GPAA.

\section{ABSTRACT}

Purpose: To evaluate the frequency, location and pattern of visual field defects as measured by SITA strategy in primary open angle glaucoma (POAG). Methods: Six thousand and two-hundred charts were reviewed. One hundred and fifty two patients who fulfilled the inclusion criteria whe included. They were stratified according to severity into early, moderate and severe. The following visual field defects were defined: nasal step, paracentral escotomata, arcuate defect, Seidel escotomata, temporal defect, general reduction of sensitivity, anular scotomata and central island. Each examination was divided into sectors corresponding to optic disc regions. Student's $t$ test was used to compare differences in frequency. Results: In the early glaucoma group, the nasal superior step was the most frequent visual field defect, followed by paracentral scotoma and inferior nasal step. General reduction of sensitivity was noted in $7.8 \%$. Defects were more common in the superior hemifield $(\mathrm{p}<0.05)$. Conclusion: The superior nasal step and paracentral scotoma were the most comum visual field defects in early primary open angle glaucoma as evalua- ted by SITA strategy, and the superior hemifield comprises most of the defects. General reduction of sensitivity was an early and rare visual field defect.

Keywords: Glaucoma/diagnosis; Perimetry/methods; Sensory thresholds; Visual fields; Sensitivity and specificity

\section{REFERÊNCIAS}

1. Chen YF, Wang TH, Hung PT. Automated perimetry in primary open-angle glaucoma. J Formos Med Assoc. 1997;96(6):441-5.

2. Mikelberg FS, Drance MS. Glaucomatous visual field defects. In: Ritch R, Shields MB, Krupin T. The glaucomas. 2a ed. St. Louis, Mosby; 1996. vol 2, p.523-37.

3. Quigley HA, Dunkelberger GR, Green WR. Chronic human glaucoma causing selectively greater loss of large optic nerve fibers. Ophthalmology. 1988;95(3): 357-63.

4. Sommer A, Katz J, Quigley HA, Miller NR, Robin AL, Richter RC, et al. Clinically detectable nerve fiber atrophy precedes the onset of glaucomatous field loss. Arch Ophthalmol. 1991;109(1):77-83.

5. Quigley HA, Addicks EM, Green WR. Optic nerve damage in human glaucoma. III. Quantitative correlation of nerve fiber loss and visual field defect in glaucoma, ischemic neuropathy, papilledema, and toxic neuropathy. Arch Ophthalmol. 1982;100(1):135-46.

6. Hart WM Jr, Becker B. The onset and evolution of glaucomatous visual field defects. Ophthalmology. 1982;89(3):268-79.

7. Werner EB, Drance SM. Early visual field disturbances in glaucoma. Arch Ophthalmol. 1977;95(7):1173-5.

8. Delgado MF, Nguyen NT, Cox TA, Singh K, Lee DA, Fechtner RD, et al. American Academy of Ophthalmology. Ophthalmic Technology Assessment Committee 2001-2002 Glaucoma Panel. Automated perimetry: a report by the American Academy of Ophthalmology. Ophthalmology. 2002;109(12):2362-74.

9. Bengtsson B, Olsson J, Heijl A, Rootzén H. A new generation of algorithms for computerized threshold perimetry, SITA. Acta Ophthalmol Scand. 1997;75 (4):368-75.

10. Bengtsson B, Heijl A. Evaluation of a new perimetric threshold strategy, SITA, in patients with manifest and suspect glaucoma. Acta Ophthalmol Scand. 1998; 76(3):268-72.

11. Sekhar GC, Naduvilath TJ, Lakkai M, Jayakumar AJ, Pandi GT, Mandal AK, et al. Sensitivity of Swedish interactive threshold algorithm compared with standard full threshold algorithm in Humphrey visual field testing. Ophthalmology. 2000;107(7):1303-8.

12. Budenz DL, Rhee P, Feuer WJ, McSoley J, Johnson CA, Anderson DR. Sensitivity and specificity of the Swedish interactive threshold algorithm for glaucomatous visual field defects. Ophthalmology. 2002;109(6):1052-8.

13. Blumenthal EZ, Sapir-Pichhadze R. Misleading statistical calculations in faradvanced glaucomatous visual field loss. Ophthalmology. 2003;110(1): 196-200.

14. Hodapp E, Parrish RK, Anderson DR. Clinical decisions in glaucoma. St. Louis: Mosby; 1993. p.52-61.

15. Garway-Heath DF, Poinoosawmy D, Fitzke FW, Hitchings RA. Mapping the visual field to the optic disc in normal tension glaucoma eyes. Ophthalmology. 2000;107(10):1809-15.

16. Advanced Glaucoma Intervention Study. 2. Visual field test scoring and reliability. Ophthalmology. 1994;101(8):1445-55.

17. Gramer E, Gerlach R, Krieglstein GK, Leydhecker W. [Topography of early glaucomatous visual field defects in computerized perimetry]. Klin Monatsbl Augenheilkd. 1982;180(6):515-23. German.

18. Asman P, Heijl A. Diffuse visual field loss and glaucoma. Acta Ophthalmol(Copenh). 1994;72(3):303-8.

19. Jonas JB, Budde WM. Is the nasal optic disc sector important for morphometric glaucoma diagnosis? Br J Ophthalmol. 2002;86(11):1232-5.

20. Nicholas SP, Werner EB. Location of early glaucomatous visual field defects. Can J Ophthalmol. 1980;15(3):131-3. 